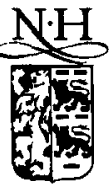

ELSEVIER

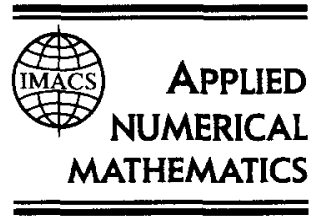

\title{
On the supraconvergence of elliptic finite difference schemes
}

\author{
J.A. Ferreira ${ }^{a, *}$, R.D. Grigorieff ${ }^{b}, 1$ \\ ${ }^{a}$ Universidade de Coimbra, Faculdade de Ciências e Tecnologia, Departamento de Matemática, Apartado 3008, \\ 3000 Coimbra, Portugal \\ ${ }^{\mathrm{b}}$ Technische Universität Berlin, Straße d. 17. Juni 135, D-10623 Berlin, Germany
}

Received 2 September 1997; received in revised form 19 November 1997; accepted 6 January 1998

\begin{abstract}
This paper deals with the supraconvergence of elliptic finite difference schemes on variable grids for second order elliptic boundary value problems subject to Dirichlet boundary conditions in two-dimensional domains. The assumptions in this paper are less restrictive than those considered so far in the literaturc allowing also variable coefficients, mixed derivatives and polygonal domains. The nonequidistant grids we consider are more flexible than merely rectangular ones such that, e.g., local grid refinements are covered.

The results also develop a close relation between supraconvergent finite difference schemes and piecewise linear finite element methods. It turns out that the finite difference equation is a certain nonstandard finite element scheme on triangular grids combined with a special form of quadrature. In extension to what is known for the standard finite element scheme, here also the gradient is shown to be convergent of second order, and so our result is also a superconvergence result for the underlying finite element method. 1998 Elsevier Science B.V. and IMACS. All rights reserved.
\end{abstract}

Keywords: Nonuniform grids; Finite difference scheme; Stability; Supraconvergence; Superconvergence

\section{Introduction}

In studying finite difference schemes for BVODEs on nonuniform grids the so-called supraconvergence was found (see [12]): although the truncation error for many natural schemes is pointwise of order one only the finite difference solutions are second order convergent. Supraconvergence was then also established for finite difference approximations for the Laplacian in a rectangular region (see $[3,11,13]$ ) and for one-dimensional parabolic equations (see [11]).

In the present paper we consider the supraconvergence of the finite difference scheme (4) for second order elliptic boundary value problems

\footnotetext{
* Corresponding author. E-mail: ferreira@mat.uc.pt.

${ }^{1}$ E-mail: grigo@math.tu-berlin.de.
} 


$$
A u:=-\left(a u_{x}\right)_{x}-\left(b u_{x}\right)_{y}-\left(b u_{y}\right)_{x}-\left(c u_{y}\right)_{y}+d u_{x}+e u_{y}+f u=g \quad \text { in } \Omega \subset \mathbb{R}^{2}
$$

subject to Dirichlet boundary conditions in polygonal regions.

Our aim is twofold. On the one hand, we prove second order supraconvergence for (4) under less restrictive assumptions considered so far in that we admit problems with variable coefficients which are allowed to have mixed derivatives and which are not assumed to be strongly coercive but only uniquely solvable. Also the domains are only assumed to be polygonal (except in $[1,2]$ ), which is somewhat more adequate than the rectangular shaped regions considered so far.

We also analyze schemes which are obtained by adding new grid lines and consequently gridpoints. Here the additional assumption is imposed that each rectangle where a new gridline starts does not become too small compared to its length. The resulting finite difference approximation is not pointwise consistent in the gridpoint, where the gridline starts and in some neighbor gridpoints. We prove the supraconvergence result (see Theorem 3 ) that the convergence order is $\mathrm{O}\left(H_{\max }^{2}+H_{\max } V^{1 / 2}\right)$, where $V$ is the total area of the rectangles where additional gridlines start and $H_{\max }$ denotes the maximal mesh-size. If there are only few of such rectangles or if the total area $V$ is of order $\mathrm{O}\left(H_{\max }^{2}\right)$, which, e.g., holds true in the case of a refinement near an isolated singularity, second order convergence for solution and gradient is obtained.

On the other hand, our results develop the close relation of the supraconvergent finite difference scheme to a certain finite element method. From this point of view our finite difference scheme is nothing else than a nonstandard piecewise linear finite element approximation on a triangular grid combined with a special form of quadrature. A numerical implementation of the scheme (4) can be based on this relation. We show in our main theorem (Theorem 1) that-in contrary to what is known for the standard finite element method-the gradient is also second order convergent. So our result, expressed in the language of finite element methods, is a superconvergence result. Since there is no restriction on the nonuniformity of the grids the triangles are allowed to have arbitrary small angles, i.e., the corresponding triangulation needs not to be quasi-uniform. Already about two decades ago Zlámal (see $[10,18]$ ) has found the superconvergence of the gradient for certain quadrature finite element solutions on nearly rectangular grids. His result also applies to higher order continuous elements but does not include our scheme.

Exploiting the relation to finite element methods clarifies and simplifies the analysis of supraconvergence. In the literature there have been developed so far different ways to treat supraconvergence. In the one-dimensional case, where naturally quite general schemes can be analyzed with reasonable effort, the analysis given in $[9,11,12]$ is via a transformation of the finite difference scheme approximating second order equations into a centered scheme for an equivalent first order system. The idea in [8] is to use an explicit representation of the discrete Green's function for the second order central divided difference operator. Another approach was undertaken in [6], where stability and order of the truncation error were considered with respect to negative norms which can be represented in the one-dimensional case in the form of the so-called Spijker norms.

The analysis of the present paper is also based on using negative norms. That this is an appropriate way of analysis was already observed in [13]. Supraconvergence results for certain elliptic finite difference schemes similar to those considered in this paper using the concept of negative norms were proved by the first author in his Ph.D. thesis [1] and in [2]. Still another approach was carried out in [3] for elliptic equations of the form (1) with $b=d=e=0$, where a grid function of order two in the mesh-size is constructed such that the finite difference operator applied to this function represents exactly the first order part in the truncation error. With the aid of the maximum principle then second order convergence 
in the maximum norm is shown. The supraconvergence results obtained in $[4,5,11]$ concern problems with one space variable only. Supraconvergence for hyperbolic problems is studied in [14,17].

All the results obtained can also be proved along the same lines for $n$-dimensional regions, $n \geqslant 3$.

One drawback of the finite difference scheme we consider in this paper is the smoothness assumption of the solution of (1), in general $u \in C^{4}(\bar{\Omega})$. More competitive in this respect are finite volume schemes, where also supraconvergence has been established (see $[9,12]$ for $n=1$ and $[16,15]$ for $n=2$ ).

\section{The finite difference scheme}

Let $\Omega \subset \mathbb{R}^{2}$ be a bounded polygonal domain, i.e., the boundary $\partial \Omega$ of $\Omega$ is the union of straight line segments. We consider the solution of (1) with zero Dirichlet boundary conditions. The variational formulation of this problem is:

find $u \in H_{0}^{1}(\Omega)$ such that

$$
a(u, v)=(g, v), \quad v \in H_{0}^{1}(\Omega) .
$$

Here $H_{0}^{1}(\Omega)$ denotes the usual $L^{2}$ Sobolev space with zero boundary conditions, $(\cdot, \cdot)$ is the standard inner product in $L^{2}(\Omega)$ and $a(\cdot, \cdot)$ is the standard sesquilinear form associated with the elliptic operator $A$. We also use the notation $\|\cdot\|_{1}$ for the usual norm in $H_{0}^{1}(\Omega)$. The coefficients of $A$ are assumed to be smooth enough, e.g., $a, b, c \in C^{3}(\bar{\Omega}), d, e \in C^{\prime}(\bar{\Omega}), f \in C(\bar{\Omega})$ is sufficient. We also impose the general assumption that the homogeneous problem (2), i.e., with $g$ taken to be equal to zero, has the solution $u=0$ only.

Let $h=\left(h_{j}\right)_{\mathbb{Z}}$ and $k=\left(k_{\ell}\right)_{\mathbb{Z}}$ be two sequences of positive numbers. We define the grid

$$
\mathbb{R}_{1}=\left\{x_{j} \in \mathbb{R}: x_{j+1}=x_{j}+h_{j}, j \in \mathbb{Z}\right\}
$$

with $x_{0} \in \mathbb{R}$ given and a corresponding grid $\mathbb{R}_{2}$ with the mesh-size vector $k$ in place of $h$. Let

$$
\mathbb{R}_{H}=\mathbb{R}_{1} \times \mathbb{R}_{2} \subset \mathbb{R}^{2} .
$$

Define also

$$
\Omega_{H}:=\Omega \cap \mathbb{R}_{H}, \quad \partial \Omega_{H}:=\partial \Omega \cap \mathbb{R}_{H}, \quad \bar{\Omega}_{H}=\bar{\Omega} \cap \mathbb{R}_{H} .
$$

The grid $\bar{\Omega}_{H}$ is assumed to satisfy the following regularity condition with respect to the region $\Omega$.

(Reg) Let $\square$ denote any subrectangle $\left(x_{j}, x_{j+1}\right) \times\left(y_{\ell}, y_{\ell+1}\right)$ formed by the grid $\mathbb{R}_{H}$. Then either $\square \cap \partial \Omega$ is empty or it is a diagonal of $\square$. In the case that the operator $A$ contains mixed derivatives then always $\square \cap \partial \Omega=\emptyset$.

The second case in condition (Reg) means that in the case $b \neq 0$ the boundary $\partial \Omega$ contains no oblique sections (but see Remark 2).

We are now preparing the definition of the finite difference approximation $A_{H} u_{H}$ of (1). For each grid point $\left(x_{j}, y_{\ell}\right) \in \mathbb{R}_{H}$ we define the central finite difference quotients

$$
\delta_{x}^{(1 / 2)} w_{j, \ell}=\frac{w_{j+1 / 2, \ell}-w_{j-1 / 2, \ell}}{x_{j+1 / 2}-x_{j-1 / 2}}, \quad \delta_{x}^{(1 / 2)} w_{j+1 / 2, \ell}=\frac{w_{j+1, \ell}-w_{j, \ell}}{x_{j+1}-x_{j}}, \quad \delta_{x} w_{j, \ell}=\frac{w_{j+1, \ell}-w_{j-1, \ell}}{x_{j+1}-x_{j-1}},
$$

where $x_{j+1 / 2}:=x_{j}+h_{j} / 2, x_{j-1 / 2}:=x_{j}-h_{j-1} / 2$. Correspondingly, the central finite difference quotients with respect to the variable $y$ are defined. 
By $\stackrel{\circ}{W}_{H}$ we denote the space of grid functions $v_{H}$ defined on $\bar{\Omega}_{H}$ with zero boundary conditions, i.e., satisfying

$$
v_{H}\left(x_{j}, y_{\ell}\right)=0, \quad\left(x_{j}, y_{\ell}\right) \in \partial \Omega_{H} .
$$

The discretized problem then reads as follows:

find $u_{H} \in \stackrel{\circ}{W}_{H}$ such that

$$
\begin{aligned}
A_{H} u_{H}:= & -\delta_{x}^{(1 / 2)}\left(a \delta_{x}^{(1 / 2)} u_{H}\right)-\delta_{x}\left(b \delta_{y} u_{H}\right)-\delta_{y}\left(b \delta_{x} u_{H}\right)-\delta_{y}^{(1 / 2)}\left(c \delta_{y}^{(1 / 2)} u_{H}\right) \\
& +d \delta_{x} u_{H}+e \delta_{y} u_{H}+f u_{H}=g \text { in } \Omega_{H} .
\end{aligned}
$$

Let $R_{H} v$ be the pointwise restriction of a function $v$ to the grid $\bar{\Omega}_{H}$. By Taylor expansion it is seen that even for a smooth solution $u$ the truncation error

$$
T_{H}:=A_{H} R_{H} u-R_{H} g
$$

is on a nonuniform grid in general only of first order pointwise consistent. Nevertheless we will prove second order convergence as stated in the theorem below, a fact which is called supraconvergence.

Let $\mathcal{T}_{H}$ be any triangulation of $\bar{\Omega}$ such that the nodes of $\mathcal{T}_{H}$ coincide with $\bar{\Omega}_{H}$. By $P_{H} v_{H}$ we denote the continuous piecewise linear interpolation of a grid function $v_{H}$ with respect to $\mathcal{T}_{H}$. We write $\|\cdot\|_{r}$ for the standard norm in the Sobolev space $H^{r}(\Omega), r \in \mathbb{N}_{0}$, and $\|\cdot\|_{r, \Delta}$ if the underlying region is the triangle $\Delta$. The notation $\|\cdot\|_{r, \infty}$ is used for the standard norm in $W^{r, \infty}(\Omega)$. By $H_{\max }$ we denote the maximal mesh-size in both $x$ - and $y$-direction.

Theorem 1. Assume that the grids $\bar{\Omega}_{H}$ satisfy condition (Reg). Let the variational problem (2) be uniquely solvable and assume that the solution $u$ of $(1)$ is in $C^{4}(\bar{\Omega})$. Then, for $H_{\max }$ small enough, the finite difference scheme (4) has a unique solution $u_{H} \in \stackrel{\circ}{W}_{H}$ satisfying the error estimate

$$
\left\|P_{H} u_{H}-P_{H} R_{H} u\right\|_{1} \leqslant C\left[\sum_{\Delta \in \mathcal{T}_{I I}}|\Delta|(\operatorname{diam} \Delta)^{4}\|u\|_{4, \infty, \Delta}^{2}\right]^{1 / 2} .
$$

Here and in the following $C$ denotes a generic constant independent of the significant quantities. We have used the notation $|\Delta|$ for the area of $\Delta$.

From the estimate (5) second order convergence follows immediately, but more precisely it also reflects the contributions of the local error to the global one.

\section{Variational formulation}

The aim of this section is to write the finite difference scheme in the form of a variational problem: find $u_{H} \in \stackrel{\circ}{W}_{H}$ such that

$$
a_{H}\left(u_{H}, v_{H}\right)=\left(R_{H} g, v_{H}\right)_{H}, \quad v_{H} \in \stackrel{\circ}{W}_{H} .
$$

Here

$$
\left(v_{H}, w_{H}\right)_{H}:=\sum_{\left(x_{j}, y_{\ell}\right) \in \Omega_{H}} \omega_{j, \ell} v_{j, \ell} \bar{w}_{j, \ell}
$$


is an inner product on $\stackrel{\circ}{W}_{H}$, where the weights $\omega_{j, \ell}$ are given by

$$
\omega_{j, \ell}:=\frac{h_{j-1}+h_{j}}{2} \frac{k_{\ell-1}+k_{\ell}}{2}, \quad\left(x_{j}, y_{\ell}\right) \in \Omega_{H} \text {. }
$$

It will be shown in the following how the sesquilinear form $a_{H}(\cdot, \cdot)$ is related to the form $a(\cdot, \cdot)$ restricted to continuous piecewise linear elements on a certain triangulation $\mathcal{T}_{H}$ with vertices equal to the set $\bar{\Omega}_{H}$. It is well known that, e.g., the five-point approximation of the Laplacian in terms of the nodal variables can be obtained in this way. In our more general case some complications arise due to the presence of the mixed derivative term. For example it is readily seen that the finite difference scheme corresponding to the standard finite element equation for piecewise linear elements corresponds to a finite difference scheme which is not even pointwise consistent if $A$ contains mixed derivative terms.

To give the definition of $a_{H}(\cdot, \cdot)$ we consider two special triangulations related to the set $\bar{\Omega}_{H}$, which we call $\mathcal{T}_{H}^{(1)}$ and $\mathcal{T}_{H}^{(2)}$. They are obtained from the disjoint decomposition

$$
\mathbb{R}_{H}=\mathbb{R}_{H}^{(1)} \dot{\cup} \mathbb{R}_{H}^{(2)}
$$

where the sum $j+\ell$ of the indices of the points $\left(x_{j}, y_{\ell}\right)$ in $\mathbb{R}_{H}^{(1)}$ and in $\mathbb{R}_{H}^{(2)}$ is even or odd, respectively. To simplify the following definition we introduce $\mathbb{R}_{H}^{(3)}:=\mathbb{R}_{H}^{(1)}$. With each point $\left(x_{j}, y_{\ell}\right) \in \mathbb{R}_{H}$ we associate the triangles $\Delta_{j, \ell}^{(i)}, i=1,2,3,4$, which have a right angle at $\left(x_{j}, y_{\ell}\right)$ and two of the four closest neighbor grid points of $\left(x_{j}, y_{\ell}\right)$ as further vertices. We then define the triangulations

$$
\begin{aligned}
& \mathcal{T}_{H, 1}^{(s)}:=\left\{\Delta_{j, \ell}^{(i)} \subset \bar{\Omega},\left(x_{j}, y_{\ell}\right) \in \mathbb{R}_{H}^{(s)}, i \in\{1,2,3,4\}\right\}, \\
& \mathcal{T}_{H, 2}^{(s)}:=\left\{\Delta_{j, \ell}^{(i)} \subset\left(\bar{\Omega} \backslash \bigcup_{\Delta \in \mathcal{T}_{H, 1}^{(s)}} \stackrel{\circ}{\Delta}\right),\left(x_{j}, y_{\ell}\right) \in \mathbb{R}_{H}^{(s+1)}, i \in\{1,2,3,4\}\right\}, \\
& \mathcal{T}_{H}^{(s)}:=\mathcal{T}_{H .1}^{(s)} \cup \mathcal{T}_{H, 2}^{(s)}, \quad s=1,2,
\end{aligned}
$$

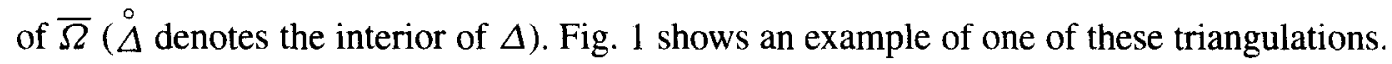

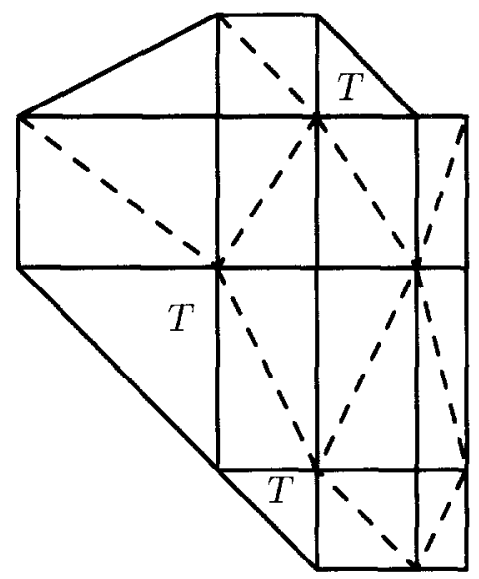

Fig. 1. Triangulation $\mathcal{T}_{H}^{(s)} . T$ indicates triangles of $\mathcal{T}_{H, 2}^{(s)}$. 
With respect to these triangulations the continuous piecewise linear interpolations $P_{H}^{(s)} v_{H}$ of $v_{H} \in \stackrel{\circ}{W}_{H}$, $s=1,2$, are well-defined.

Now we define the sesquilinear form $a_{H}(\cdot, \cdot)$ in $(6)$ as the arithmetical mean

$$
a_{H}=\frac{1}{2}\left(a_{H}^{(1)}+a_{H}^{(2)}\right)
$$

of two scsquilincar forms, each of which has the form

$$
a_{H}^{(s)}=a^{(s)}+b^{(s)}+c^{(s)}+d^{(s)}+e^{(s)}+f^{(s)}, \quad s=1,2 .
$$

The sesquilinear forms on the right-hand side of (11) are all constructed in a similar way by summing particular approximations of the "energy" related to each corresponding differential term over the triangles of $\mathcal{T}_{H}^{(s)}$. So let $\Delta \in \mathcal{T}_{H}^{(s)}$. We define $a_{\Delta}$ to be the value of $a$ in the midpoint of the side of $\Delta$ parallel to the $x$-axis. Then let

$$
a^{(s)}\left(v_{H}, w_{H}\right):=\sum_{\Delta \in \mathcal{T}_{H}^{(s)}} a_{\Delta} \int_{\Delta}\left(P_{H}^{(s)} v_{H}\right)_{x}\left(P_{H}^{(s)} \bar{w}_{H}\right)_{x} \mathrm{~d} x \mathrm{~d} y .
$$

Similarly, let $c_{\Delta}$ be the value of $c$ in the midpoint of the side of $\Delta$ parallel to the $y$-axis and

$$
c^{(s)}\left(v_{H}, w_{H}\right):=\sum_{\Delta \in \mathcal{T}_{I I}^{(s)}} c_{\Delta} \int_{\Delta}\left(P_{H}^{(s)} v_{H}\right)_{y}\left(P_{H}^{(s)} \bar{w}_{H}\right)_{y} \mathrm{~d} x \mathrm{~d} y
$$

In the approximation of the mixed derivative terms we need

$$
b_{\Delta}:=b\left(x_{\Delta}, y_{\Delta}\right) \text {, }
$$

where $\left(x_{\Delta}, y_{\Delta}\right)$ is the vertex of $\Delta$ associated with the angle $\pi / 2$ of $\Delta$. Then

$$
b^{(s)}\left(v_{H}, w_{H}\right):=\sum_{\Delta \in \mathcal{T}_{H}^{(s)}} b_{\Delta} \int_{\Delta}\left[\left(P_{H}^{(s)} v_{H}\right)_{x}\left(P_{H}^{(s)} \bar{w}_{H}\right)_{y}+\left(P_{H}^{(s)} v_{H}\right)_{y}\left(P_{H}^{(s)} \bar{w}_{H}\right)_{x}\right] \mathrm{d} x \mathrm{~d} y .
$$

For approximating the first order terms let

$$
\left(P_{H}^{(s)} v_{H}\right)_{\Delta, x}:=P_{H}^{(s)} v_{H}\left(x_{\Delta}, y_{\Delta}\right), \quad \Delta \in \mathcal{T}_{H}^{(s)},
$$

where $\left(x_{\Delta}, y_{\Delta}\right)$ is the midpoint of the side of $\Delta$ parallel to the $x$-axis. Correspondingly, we introduce $\left(P_{H}^{(s)} v_{H}\right)_{\Delta, y}$, where in this case $\left(x_{\Delta}, y_{\Delta}\right)$ is taken to be the midpoint of the side of $\Delta$ parallel to the $y$-axis. Then we define

$$
\begin{aligned}
d^{(s)}\left(v_{H}, w_{H}\right) & :=\sum_{\Delta \in \mathcal{T}_{H}^{(s)}}\left[P_{H}^{(s)}\left(d \bar{w}_{H}\right)\right]_{\Delta, x} \int_{\Delta}\left(P_{H}^{(s)} v_{H}\right)_{x} \mathrm{~d} x \mathrm{~d} y, \\
e^{(s)}\left(v_{H}, w_{H}\right) & :=\sum_{\Delta \in \mathcal{T}_{H}^{(s)}}\left[P_{H}^{(s)}\left(e \bar{w}_{H}\right)\right]_{\Delta, y} \int_{\Delta}\left(P_{H}^{(s)} v_{H}\right)_{y} \mathrm{~d} x \mathrm{~d} y .
\end{aligned}
$$

Finally,

$$
f^{(s)}\left(v_{H}, w_{H}\right):=\sum_{\left(x_{j}, y_{\ell}\right) \in \Omega_{H}} \omega_{j, \ell} f\left(x_{j}, y_{\ell}\right) v_{j, \ell} \bar{w}_{j, \ell} .
$$


By choosing $v_{H}$ to vanish in all but one single point in $\Omega_{H}$ it is an elementary calculation to verify that with these definitions the following proposition holds.

Proposition 1. With the sesquilinear form $a_{H}(\cdot, \cdot)$ defined by (10) and with $A_{H}$ defined by (4)

$$
a_{H}\left(v_{H}, w_{H}\right)=\left(A_{H} v_{H}, w_{H}\right)_{H}, \quad v_{H}, w_{H} \in \stackrel{\circ}{W}_{H} .
$$

Remark. If there are no mixed derivatives in (1) then it is not necessary to define $a_{H}$ as arithmetic mean (10). Any triangulation $\mathcal{T}_{H}$ and corresponding sesquilinear form would do the job.

\section{Inverse stability}

We now consider a sequence of grids $\mathbb{R}_{H}$ such that the maximal mesh-size $H_{\max }$ tends to zero. We use the symbol " $H \in \Lambda$ " to indicate the sequence of discretizations considered and write " $(H \in \Lambda)$ " for the convergence with respect to $H$ running through this sequence.

One main ingredient for the convergence analysis is the following inverse stability result which will be proved in this section.

Theorem 2. Let the grids $\bar{\Omega}_{H}$ satisfy condition (Reg). Assume that the coefficients of $A$ in (1) are in $C(\bar{\Omega})$ and that the homogeneous variational problem (2), i.e., with $g=0$, has only the solution $u=0$. For each $H \in \Lambda$ let $\mathcal{T}_{H}$ be a triangulation of $\Omega$. Denote by $P_{H}$ the corresponding piecewise linear interpolation operator. Then there exists a constant $C$ such that for $H \in \Lambda$ with $H_{\max }$ small enough

$$
\left\|P_{H} v_{H}\right\|_{1} \leqslant C \sup _{\substack{0 \neq w_{H} \in \dot{W}_{H}\\}} \frac{\left|a_{H}\left(v_{H}, w_{H}\right)\right|}{\left\|P_{H} w_{H}\right\|_{1}}, \quad v_{H} \in \stackrel{\circ}{W}_{H} .
$$

The estimate (20) can be given an alternative form. In the finite dimensional space $\stackrel{\circ}{W}_{H}$ of grid functions a norm is defined by setting

$$
\left|v_{H}\right|_{1, H}:=\left\|P_{H} v_{H}\right\|_{1}, \quad v_{H} \in \stackrel{\circ}{W}_{H},
$$

which is a discrete analogue of the Sobolev norm $\|\cdot\|_{1}$. Then

$$
\left|v_{H}\right|_{-1 . H}:=\sup _{\substack{0 \neq w_{H} \in \mathscr{W}_{H}\\}} \frac{\left|\left(v_{H}, w_{H}\right)_{H}\right|}{\left|w_{H}\right|_{1, H}}, \quad v_{H} \in \stackrel{\circ}{W}_{H},
$$

can be considered as a discrete analogue of the "negative" norm $\|\cdot\|_{-1}$ for functions in $L^{2}(\Omega)$. With this definition and recalling Proposition 1 the estimate (20) is equivalent to

$$
\left|v_{H}\right|_{1, H} \leqslant C\left|A_{H} v_{H}\right|_{-1, H}, \quad v_{H} \in \stackrel{\circ}{W}_{H}, H \in \Lambda .
$$

This estimate shows that the mapping $A_{H}$ is invertible for $H$ in a final section $\Lambda_{1} \subset \Lambda$ and that the sequence $A_{H}^{-1}, H \in \Lambda_{1}$, is stable, i.e., $A_{H}^{-1}$ considered as a mapping from $\left(\stackrel{\circ}{W}_{H},\left|v_{H}\right|_{-1, H}\right)$ in $\left(\stackrel{\circ}{W},\left|v_{H}\right|_{1, H}\right)$ is uniformly bounded with respect to $H \in \Lambda$. 
The proof of Theorem 2 is based on the following well-known result from finite element theory (see, e.g., [7, Theorem 8.2.8]).

Proposition 2. Under the assumptions of Theorem 2 the following estimate holds for $H \in \Lambda$ with $H_{\max }$ small enough:

$$
\left\|P_{H} v_{H}\right\|_{1} \leqslant C \sup _{\substack{0 \neq w_{H} \in \stackrel{\circ}{W}_{H}}} \frac{\left|a\left(P_{H} v_{H}, P_{H} w_{H}\right)\right|}{\left\|P_{H} w_{H}\right\|_{1}}, \quad v_{H} \in \stackrel{\circ}{W_{H}}
$$

Lemma 1. Let $s \in\{1,2\}$ and let $v_{H}, w_{H} \in \stackrel{\circ}{W}_{H}, H \in \Lambda$, be two sequences satisfying

$$
\left\|P_{H}^{(s)} v_{H}\right\|_{1} \leqslant 1, \quad\left\|P_{H}^{(s)} w_{I I}\right\|_{1} \leqslant 1, \quad H \in \Lambda .
$$

Then (see (12))

$$
a^{(s)}\left(v_{H}, w_{H}\right)-\left(a\left(P_{H}^{(s)} v_{H}\right)_{x},\left(P_{H}^{(s)} w_{H}\right)_{x}\right)_{0} \longrightarrow 0 \quad(H \in \Lambda)
$$

and also the corresponding relations for the sesquilinear forms $b^{(s)}$ and $c^{(s)}$ (see (13), (15)) hold.

Proof. Consider a triangle $\Delta \in \mathcal{T}_{H}^{(s)}$. Since the coefficient $a$ is continuous in $\bar{\Omega}$ we have

$$
a_{\Delta} \int_{\Delta}\left(P_{H}^{(s)} v_{H}\right)_{x}\left(P_{H}^{(s)} \bar{w}_{H}\right)_{x} \mathrm{~d} x \mathrm{~d} y-\int_{\Delta} a(x, y)\left(P_{H}^{(s)} v_{H}\right)_{x}\left(P_{H}^{(s)} \bar{w}_{H}\right)_{x} \mathrm{~d} x \mathrm{~d} y \longrightarrow 0 \quad(H \in \Lambda)
$$

uniformly with respect to $\Delta$, and this proves the assertion for $a^{(s)}$. The proof for $b^{(s)}$ and $c^{(s)}$ is similar.

Lemma 2. Let $\mathcal{T}_{H}$ be a triangulation of $\Omega$. For $v_{H} \in \stackrel{\circ}{W}_{H}$ and $\Delta \in \mathcal{T}_{H}$ we denote by $v_{\Delta, 1}, v_{\Delta, 2}$ and $v_{\Delta, 3}$ the values of $v_{H}$ in the vertices of $\Delta$. Then

$$
\sum_{\Delta \in \mathcal{T}_{H}}|\Delta|\left(\left|v_{\Delta, 1}\right|^{2}+\left|v_{\Delta, 2}\right|^{2}+\left|v_{\Delta, 3}\right|^{2}\right) \leqslant C\left\|P_{H} v_{H}\right\|_{0}^{2}, \quad v_{H} \in \stackrel{\circ}{W}_{H}
$$

Proof. If the assertion is known to hold for the special case $\Omega=\Delta$ with $C$ independent of $\Delta$ then it follows for a general $\Omega$ by summation. The proof for the case $\Omega=\Delta$ is obtained by performing a change of variables which maps $\Delta$ onto the unit triangle and then using the equivalence of all norms on a three-dimensional vector space.

Lemma 3. Let $s \in\{1,2\}$ and let $v_{H}, w_{H} \in \stackrel{\circ}{W}_{H}, H \in \Lambda$, be two sequences such that (22) holds. Then (see (17), (18))

$$
\begin{array}{ll}
d^{(s)}\left(v_{H}, w_{H}\right)-\left(d\left(P_{H}^{(s)} v_{H}\right)_{x}, P_{H}^{(s)} w_{H}\right)_{0} \rightarrow 0 & (H \in \Lambda), \\
e^{(s)}\left(v_{H}, w_{H}\right)-\left(e\left(P_{H}^{(s)} v_{H}\right)_{y}, P_{H}^{(s)} w_{H}\right)_{0} \longrightarrow 0 & (H \in \Lambda) .
\end{array}
$$

Proof. The two convergence relations are proved similarly and we show the last one only. Since the imbedding of $H_{0}^{1}(\Omega)$ in $L^{2}(\Omega)$ is compact and bounded sequences in $H_{0}^{1}(\Omega)$ are weakly compact, we 
can assume for the proof that $\left\{P_{H}^{(s)} w_{H}\right\}_{A}$ is strongly convergent in $L^{2}(\Omega)$ and $\left\{P_{H}^{(s)} v_{H}\right\}_{\Lambda}$ is weakly convergent in $H_{0}^{1}(\Omega)$. Let $v$ and $w \in H_{0}^{1}(\Omega)$ be the limits of $\left\{P_{H}^{(s)} v_{H}\right\}_{\Lambda}$ and $\left\{P_{H}^{(s)} w_{H}\right\}_{A}$, respectively. It follows that

$$
\left(e\left(P_{H}^{(s)} v_{H}\right)_{y}, P_{H}^{(s)} w_{H}\right)_{0} \longrightarrow\left(e u_{y}, v\right)_{0} \quad(H \in \Lambda) .
$$

We show that also

$$
e^{(s)}\left(v_{H}, w_{H}\right) \longrightarrow\left(e v_{y}, w\right)_{0} \quad(H \in \Lambda)
$$

With the aid of Lemma 2 we obtain

$$
\begin{aligned}
\left|e^{(s)}\left(v_{H}, w_{H}\right)\right|^{2} \leqslant & C \sum_{\Delta \in \mathcal{T}_{H}^{(s)}} \int_{\Delta}\left|\left(P_{H}^{(s)} v_{H}\right)_{y}\right|^{2} \mathrm{~d} x \mathrm{~d} y \\
& \times \sum_{\Delta \in \mathcal{T}_{H}^{(s)}}|\Delta|\left(\left|\left(P_{H}^{(s)} w_{H}\right)_{\Delta, 1}\right|^{2}+\left|\left(P_{H}^{(s)} w_{H}\right)_{\Delta, 2}\right|^{2}+\left|\left(P_{H}^{(s)} w_{H}\right)_{\Delta, 3}\right|^{2}\right) \\
\leqslant & C\left\|\left(P_{H}^{(s)} v_{H}\right)_{y}\right\|_{0}^{2}\left\|P_{H}^{(s)} w_{H}\right\|_{0}^{2} .
\end{aligned}
$$

Let $\phi \in C_{0}^{\infty}(\Omega)$, i.e., $\phi$ in a dense subset of $L^{2}(\Omega)$. Let $R_{H} w$ denote the pointwise restriction of a function $w$ to the grid $\bar{\Omega}_{H}$. Then

$$
P_{H}^{(s)} R_{H} \phi \longrightarrow \phi \quad \text { in } L^{2}(\Omega) \quad(h \in \Lambda)
$$

and one concludes with the aid of (24) that for proving (23) it is sufficient to show

$$
e^{(s)}\left(v_{H}, R_{H} \phi\right) \longrightarrow\left(e v_{y}, \phi\right)_{0} \quad(H \in \Lambda) .
$$

This is indeed the case since the piecewise constant function $\psi_{H}$ defined by

$$
\psi_{H}(x, y):=\left[P_{H}^{(s)}\left(e R_{I I} \phi\right)\right]_{\Delta, y}, \quad(x, y) \in \Delta, \Delta \in \mathcal{T}_{H}^{(s)},
$$

is strongly convergent in $L^{2}(\Omega)$ with limit $e \phi$ and we can write

$$
e^{(s)}\left(v_{H}, R_{H} \phi\right)=\left(\left(P_{H}^{(s)} v_{H}\right)_{y}, \psi_{H}\right)_{0} .
$$

Lemma 4. Under the assumptions of Lemma 3

$$
f^{(s)}\left(v_{H}, w_{H}\right)-\left(f P_{H}^{(s)} v_{H}, P_{H}^{(s)} w_{H}\right)_{0} \longrightarrow 0 \quad(H \in \Lambda) .
$$

Proof. The same kind of reasoning as in Lemma 3 is used but this time based on the fact that for $\phi, \psi \in C_{0}^{\infty}(\Omega)$

$$
f^{(s)}\left(R_{H} \phi, R_{H} \psi\right) \longrightarrow(f \phi, \psi)_{0} \quad(H \in \Lambda) .
$$

Proof of Theorem 2. It is not difficult to see that for any two triangulations of $\Omega$ the corresponding norms $\left\|P_{H} w_{H}\right\|_{1}$ are uniformly in $H \in \Lambda$ equivalent. Hence the assertion will follow from (21) if we show that for $s \in\{1,2\}$ and any two sequences $\left\{v_{H}\right\}_{\Lambda}$ and $\left\{w_{H}\right\}_{\Lambda}$ satisfying (22) one has

$$
\left|a_{H}^{(s)}\left(v_{H}, w_{H}\right)-a\left(P_{H}^{(s)} v_{H}, P_{H}^{(s)} w_{H}\right)\right| \longrightarrow 0 \quad(H \in \Lambda) .
$$

This was proved in Lemmas 1, 3 and 4. 


\section{Truncation error estimate}

The form (20) of the stability inequality suggests to obtain an error estimate by bounding the quantity

$$
\frac{a_{H}\left(R_{H} u, v_{H}\right)-\left(R_{H} g, v_{H}\right)_{H}}{\left\|P_{H} v_{H}\right\|_{1}} .
$$

According to the definition (10), (11) of $a_{H}(\cdot, \cdot)$ we have to consider several terms. They all have a certain error structure in common: for each fixed triangle $\Delta$ in $\mathcal{T}_{H}^{(s)}$ one obtains by Taylor expansion in each vertex a contribution to the differential operator $A u$. Furthermore, there is a first order error term for which an additional power of $H_{\max }$ can be gained by splitting off the factor $\left(P_{H} v_{H}\right)_{x}$ or $\left(P_{H} v_{H}\right)_{y}$ thus exhibiting supraconvergence. The remainder is of second order.

Let $s \in\{1,2\}$ and choose a triangle $\Delta \in \mathcal{T}_{H}^{(s)}$. We assume that $\Delta$ has the vertices

$$
\left(x_{j}, y_{\ell}\right),\left(x_{j}, y_{\ell+1}\right),\left(x_{j+1}, y_{\ell}\right) \text {. }
$$

Different shapes of $\Delta$ can be handled similarly. For simplicity we write in the following only $u$ in place of $R_{H} u$. Consider

$$
T_{a}:=a_{\Delta} \int_{\Delta}\left(P_{H} u\right)_{x}\left(P_{H} \bar{v}_{H}\right)_{x} \mathrm{~d} x \mathrm{~d} y=\left(a u_{x}\right)_{j+1 / 2, \ell} \frac{\bar{v}_{j+1, \ell}-\bar{v}_{j, \ell}}{h_{j}}|\Delta|+R_{a, 1},
$$

where $|\Delta|=h_{j} k_{\ell} / 2$ and $R_{a, 1}$ satisfies the estimate

$$
\left|R_{a, 1}\right| \leqslant \frac{h_{j}^{2}}{24}\left\|a u_{x x x}\right\|_{\infty, \Delta} \int_{\Delta}\left|\left(P_{H} v_{H}\right)_{x}\right| \mathrm{d} x \mathrm{~d} y .
$$

Expanding further we obtain

$$
\left(a u_{x}\right)_{j+1 / 2, \ell}=\left(a u_{x}\right)_{j, \ell}+\frac{h_{j}}{2}\left(\left(a u_{x}\right)_{x}\right)_{j, \ell}+\frac{h_{j}^{2}}{8}\left(\left(a u_{x}\right)_{x x}\right)_{j, \ell}+R_{a, 2},
$$

where

$$
\left|R_{a, 2}\right| \leqslant \frac{h_{j}^{3}}{48}\left\|\left(a u_{x}\right)_{x x x}\right\|_{\infty, \Delta} .
$$

Using the corresponding expansion with respect to the point $\left(x_{j+1}, y_{\ell}\right)$ we derive

$$
T_{a}=\frac{k_{\ell}}{2}\left[\left(a u_{x}\right)_{j+1, \ell}-\frac{h_{j}}{2}\left(\left(a u_{x}\right)_{x}\right)_{j+1, \ell}\right] \bar{v}_{j+1, \ell}-\frac{k_{\ell}}{2}\left[\left(a u_{x}\right)_{j, \ell}+\frac{h_{j}}{2}\left(\left(a u_{x}\right)_{x}\right)_{j, \ell}\right] \bar{v}_{j, \ell}+R_{a, 3},
$$

where $R_{a, 3}$ can be estimated by

$$
\begin{aligned}
\left|R_{a, 3}\right| \leqslant & \frac{h_{j}^{2}}{24}\left(\left\|a u_{x x x}\right\|_{\infty, \Delta}+3\left\|\left(a u_{x}\right)_{x x}\right\|_{\infty, \Delta}\right) \int_{\Delta}\left|\left(P_{H} v_{H}\right)_{x}\right| \mathrm{d} x \mathrm{~d} y \\
& +\frac{h_{j}^{2}}{12}\left\|\left(a u_{x}\right)_{x x x}\right\|_{\infty, \Delta}|\Delta|\left(\left|v_{j, \ell}\right|+\left|v_{j+1, \ell}\right|\right) .
\end{aligned}
$$

Summation of (28) over all triangles $\Delta \in \mathcal{T}_{H}^{(s)}$ gives

$$
a^{(s)}\left(R_{H} u, v_{H}\right)=-\left(R_{H}\left(a u_{x}\right)_{x}, v_{H}\right)_{H}+R_{a}
$$


with $R_{a}$ satisfying

$$
\left|R_{a}\right| \leqslant C\left(\sum_{\Delta \in \mathcal{T}_{H}^{(s)}}|\Delta| h_{\Delta}^{4}\left(\left\|a u_{x x x}\right\|_{\infty, \Delta}+\left\|\left(a u_{x}\right)_{x x}\right\|_{1, \infty, \Delta}\right)^{2}\right)^{1 / 2}\left\|P_{H} v_{H}\right\|_{1},
$$

where for estimating $\sum|\Delta|\left(\left|v_{j, \ell}\right|^{2}+\left|v_{j+1, \ell}\right|^{2}\right)$ Lemma 2 was taken into account; also $v_{H}=0$ on $\partial \Omega_{H}$ was used in (28) for triangles $\Delta$ at the boundary of $\Omega$.

In the same way

$$
c^{(s)}\left(R_{H} u, v_{H}\right)=-\left(R_{H}\left(c u_{y}\right)_{y}, v_{H}\right)_{H}+R_{c}
$$

can be proved, where $R_{c}$ satisfies an estimate similar to $R_{a}$ in (30) only with $\partial / \partial x, h_{\Delta}$ and $a$ replaced by $\partial / \partial y, k_{\Delta}$ and $c$, respectively.

Next we consider the mixed derivatives term $\frac{1}{2}\left(b^{(1)}+b^{(2)}\right)$. We give only the details for the part in (15) corresponding to $\left(b u_{x}\right)_{y}$, which we denote by $b_{(x y)}^{(s)}$. We choose an arbitrary point $\left(x_{j}, y_{\ell}\right) \in \Omega_{H}$ and collect all contributions in the sesquilinear forms $b_{(x y)}^{(s)}, s=1,2$, which contain a factor $\bar{v}_{j, \ell}$. As a typical quantity we have to consider

$$
T_{b, \Delta}:=b_{\Delta} \int_{\Delta}\left(P_{H} u\right)_{x}\left(P_{H} \bar{v}_{H}\right)_{y} \mathrm{~d} x \mathrm{~d} y .
$$

In one of the triangulations $\mathcal{T}_{H}^{(s)}$ there are exactly the triangles $\Delta_{j, \ell}^{(i)}, i=1,2,3,4$, involved which have a right angle at $\left(x_{j}, y_{\ell}\right)$ (see (9)). The factor of $\bar{v}_{j, \ell}$ coming from these triangles is easily seen to be zero. In the other triangulation there are eight triangles which have $\left(x_{j}, y_{\ell}\right)$ as vertex. Obviously, four of them contribute nothing to $\bar{v}_{i, \ell}$. We consider the remaining four triangles. One of them, for example, has vertices

$$
\left(x_{j}, y_{\ell}\right),\left(x_{j}, y_{\ell-1}\right),\left(x_{j+1}, y_{\ell-1}\right) .
$$

We evaluate (32) for this triangle and determine as factor of $\bar{v}_{j, \ell}$

$$
T_{b, 1}:=\frac{1}{2} b_{j, \ell-1}\left(u_{j+1, \ell-1}-u_{j, \ell-1}\right) \text {. }
$$

By suitably expanding this expression with respect to $x$ and $y$ we obtain

$$
T_{b, 1}=\frac{1}{2} b_{j, \ell}\left(u_{j+1, \ell}-u_{j, \ell}\right)-|\Delta|\left[\left(b u_{x}\right)_{y}\right]_{j, \ell}+\frac{1}{2}|\Delta|\left[k_{\ell-1}\left(b u_{x}\right)_{y y}-h_{j}\left(b u_{x x}\right)_{y}\right]_{j, \ell}+R_{b, 1},
$$

where $R_{b, 1}$ satisfies the estimate

$$
\left|R_{b, 1}\right| \leqslant \frac{|\Delta|}{6}\left[h_{j}^{2}\left\|\left(b u_{x x x}\right)_{y}\right\|_{\infty, \Delta}+\frac{3}{2} h_{j} k_{\ell-1}\left\|\left(b u_{x x}\right)_{y y}\right\|_{\infty, \Delta}+k_{\ell-1}^{2}\left\|\left(b u_{x}\right)_{y y y}\right\|_{\infty, \Delta}\right] .
$$

Corresponding expansions hold for the other three triangles. By summation we determine the factor of $\bar{v}_{j, \ell}$ in the sesquilincar form

$$
\frac{1}{2}\left(b_{(x y)}^{(1)}+b_{(x y)}^{(2)}\right)\left(R_{H} u, v_{H}\right)
$$

to be

$$
\begin{aligned}
T_{b, 2}:= & -\left[\left(b u_{x}\right)_{y}\right]_{j, \ell} \omega_{j, \ell}+\frac{1}{4}\left(h_{j-1}+h_{j}\right)\left(k_{\ell-1}^{2}-k_{\ell}^{2}\right)\left[\left(b u_{x}\right)_{y y}\right]_{j, \ell} \\
& +\frac{1}{4}\left(k_{\ell-1}+k_{\ell}\right)\left(h_{j-1}^{2}-h_{j}^{2}\right)\left[\left(b u_{x x}\right)_{y}\right]_{j, \ell}+R_{b, 2},
\end{aligned}
$$


where $R_{b, 2}$ is the sum of the remainder terms $R_{b .1}$ belonging to the four relevant triangles adjacent to $\left(x_{j}, y_{\ell}\right)$.

As a preparation for the summation of (35) over $\Omega_{H}$ we establish for the function $w:=\left(b u_{x}\right)_{y y}$ and $v_{H}$ satisfying $v_{j, m-1}=v_{j, n+1}=0$ the identity

$$
\begin{aligned}
\sum_{\ell=m}^{n} w_{j, \ell}\left(k_{\ell-1}^{2}-k_{\ell}^{2}\right) \bar{v}_{j, \ell} & =\sum_{\ell=m-1}^{n} k_{\ell}^{2}\left[\left(w_{j, \ell+1}-w_{j, \ell}\right) \bar{v}_{j, \ell}+w_{j, \ell+1}\left(\bar{v}_{j, \ell+1}-\bar{v}_{j, \ell}\right)\right] \\
& =\sum_{\ell=m-1}^{n} k_{\ell}^{3}\left[\left(w_{y}\right)_{j, \ell+\theta} \bar{v}_{j, \ell}+w_{j, \ell+1} \frac{\bar{v}_{j, \ell+1}-\bar{v}_{j, \ell}}{k_{\ell}}\right]
\end{aligned}
$$

(the index $\ell+\theta$ indicates a point between $y_{\ell}$ and $y_{\ell+1}$ ). A similar result applies to the summation of the last term in (35) with respect to $j$. Hence, multiplication of (35) by $\bar{v}_{j, \ell}$ and summation over $\Omega_{H}$ furnishes

$$
\frac{1}{2}\left(b_{(x y)}^{(1)}+b_{(x y)}^{(2)}\right)\left(R_{H} u, v_{H}\right)=-\left(R_{H}\left(b u_{x}\right)_{y}, v_{H}\right)_{H}+R_{b},
$$

where

$$
\begin{aligned}
\left|R_{b}\right| \leqslant C[ & \sum_{\Delta \in \mathcal{T}_{H}}|\Delta|\left(h_{\Delta}^{4}+k_{\Delta}^{4}\right) \\
& \left.\times\left(\left\|\left(b u_{x}\right)_{y y}\right\|_{1, \infty, \Delta}^{2}+\left\|\left(b u_{x x}\right)_{y}\right\|_{1, \infty, \Delta}^{2}+\left\|\left(b u_{x x x}\right)_{y}\right\|_{\infty, \Delta}^{2}\right)\right]^{1 / 2}\left\|P_{H} v_{H}\right\|_{1} .
\end{aligned}
$$

For the approximation of the mixed derivative term $\left(b u_{y}\right)_{x}$ a corresponding result holds, where the remainder term can be estimated as in (38) only with the derivatives with respect to $x$ and $y$ interchanged.

We are left with the first order term. We consider the approximation of $d u_{x}$ and collect the coefficient $T_{d, 2}$ of $\bar{v}_{j, \ell}$ in the sesquilinear form $\frac{1}{2}\left(d^{(1)}+d^{(2)}\right)$. Let $\Delta$ be the triangle with vertices (27). Then

$$
T_{d, 1}:=\frac{1}{2} d_{j, \ell} \int_{\Delta}\left(P_{H}^{(s)} u\right)_{x} \mathrm{~d} x \mathrm{~d} y=\frac{k_{\ell}}{4} d_{j, \ell}\left(u_{j+1, \ell}-u_{j, \ell}\right)=\frac{k_{\ell}}{4} d_{j, \ell}\left(h_{j} u_{x}+\frac{h_{j}^{2}}{2} u_{x x}\right)_{j, \ell}+R_{d, 1},
$$

where $R_{d, 1}$ can be bounded by

$$
\left|R_{d, 1}\right| \leqslant \frac{h_{j}^{2}}{12}\left|d_{j, \ell}\right|\left\|u_{x x x}\right\|_{\infty, \Delta}|\Delta|
$$

By summing this and the corresponding results for the remaining seven triangles which contribute to $T_{d, 2}$ we calculate

$$
T_{d, 2}=d_{j, \ell}\left(u_{x}\right)_{j, \ell} \omega_{j, \ell}+d_{j, \ell}\left(u_{x x}\right)_{j, \ell}\left(h_{j}^{2}-h_{j-1}^{2}\right) \frac{k_{\ell-1}+k_{\ell}}{8}+R_{d, 2},
$$

where $R_{d, 2}$ is the sum of the remainder terms corresponding to (39). A summation over $\Omega_{H}$ yields

$$
\frac{1}{2}\left(d^{(1)}+d^{(2)}\right)\left(R_{H} u, v_{I I}\right)=\left(R_{I I}\left(d u_{x}\right), v_{H}\right)+R_{d}
$$

where

$$
\left|R_{d}\right| \leqslant C\left[\sum_{\Delta \in \mathcal{T}_{H}}|\Delta| h_{\Delta}^{4}\left(\|d\|_{\infty, \Delta}^{2}\left\|u_{x x x}\right\|_{\infty, \Delta}^{2}+\left\|\left(d u_{x x}\right)_{x}\right\|_{\infty, \Delta}^{2}\right)\right]^{1 / 2}\left\|P_{H} v_{H}\right\|_{1}
$$


A similar result holds for the discretization of $e u_{y}$. The bound for the remainder term $R_{e}$ in this case is obtained by taking $k_{\Delta}, e$ and $\partial / \partial y$ in place of $h_{\Delta}, d$ and $\partial / \partial x$, respectively, in (42).

Altogether we have proved the following

Proposition 3. Assume that the grids $\bar{\Omega}_{H}$ satisfy condition (Reg). Let $u \in C^{4}(\bar{\Omega})$. Then

$$
a_{H}\left(R_{H} u, v_{H}\right)=\left(R_{H}(A u), v_{H}\right)_{H}+\tau_{H}\left(u, v_{H}\right)
$$

with $\tau_{H}$ satisfying the estimate

$$
\left|\tau_{H}\left(u, v_{H}\right)\right| \leqslant C\left[\sum_{\Delta \in \mathcal{T}_{H}}|\Delta|(\operatorname{diam} \Delta)^{4}\left(\left\|u_{x}\right\|_{3, \infty, \Delta}^{2}+\left\|u_{y}\right\|_{3, \infty . \Delta}^{2}\right)\right]^{1 / 2}\left\|P_{H} v_{H}\right\|_{1},
$$

where the constant $C$ is dependent on the coefficients of $A$ but not on the triangulation $\mathcal{T}_{H}$ or $u$.

Remark 1. The dependence of $C$ in (43) on the coefficients of $A$ can be easily made more explicit based on the more detailed estimates for the remainder terms derived in (30), (38), (42) and the remarks following them.

Theorem 1 is an immediate consequence of Propositions 2 and 3.

Remark 2. If the boundary $\partial \Omega$ contains a section which is not parallel to the coordinate axes the finite difference approximation (4) can still be used. It is only necessary to give a meaning to the mixed derivative approximations in those points $P \in \Omega_{H}$ near oblique parts of $\partial \Omega$, where in forming $A_{H} v_{H}$ a grid point $P_{1}$ outside of $\bar{\Omega}$ is involved. This is simply done by defining

$$
v_{H}\left(P_{1}\right):=-v_{H}(P)
$$

In these exceptional points $P$ the resulting finite difference approximation is not consistent with $A$, the truncation error is only $O(1)$. Following the lines of argument leading to (38) it can be shown that the bound for $R_{b}$ now contains additional terms of the form

$$
\sum_{\Delta \in \mathcal{T}_{H .2}}|\Delta|(\operatorname{diam} \Delta)^{2}\|u\|_{4, \infty, \Delta}^{2}
$$

where (see (9))

$$
\mathcal{T}_{H, 2}:=\mathcal{T}_{H, 2}^{(1)} \cup \mathcal{T}_{H, 2}^{(2)}
$$

As a consequence, this shows again a supraconvergence phenomenon, where the proved overall convergence order is $\mathrm{O}\left(H_{\max }^{3 / 2}\right.$ ) (for that it was taken into account, that $\mathcal{T}_{H, 2}$ is contained in a strip of width $O\left(H_{\max }\right)$ around $\left.\partial \Omega\right)$.

\section{Local grid refinement}

We consider in this section more general grids than in Section 2 which are obtained by adding new gridline sections to the original grid. This process is allowed to be repeated and hence local grid refinements are feasible in this way. In the differential operator $A$ from (1) we take $b=0$, i.e., there are no mixed derivatives. The grids are assumed to satisfy condition (Reg). 


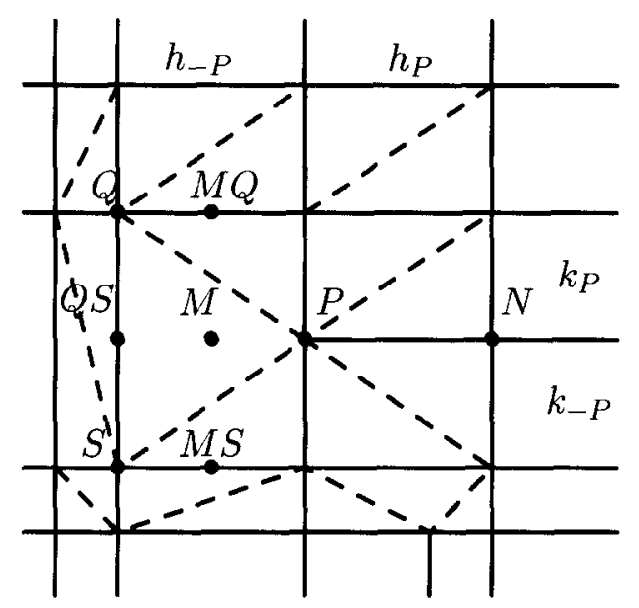

Fig. 2. New gridline section and triangulation.

For the formal description of the kind of grids we admit we start with the finest grids $\Omega_{H}$ and $\bar{\Omega}_{H}$ as defined in Section 2 which are assumed to satisfy condition (Reg) and then delete certain gridline sections. The result of this process is denoted by $\Omega_{H}^{(r)}$ and $\overline{\Omega_{H}^{(r)}}$, respectively. Let $\mathcal{T}_{H}$ be a regular triangulation of $\Omega$ such that $\Omega_{H}^{(r)}$ is the set of vertices. A triangle $\Delta \in \mathcal{T}_{H}$ is said to be in normal position if two of its sides are parallel to the coordinate axes, otherwise it is called exceptional. We assume in this section the following condition to hold.

(Rft) There exists a triangulation $\mathcal{T}_{H}$ of $\Omega$ such that each exceptional $\Delta \in \mathcal{T}_{H}$ is isosceles and exceptional triangles have no common side other than the hypotenuse perhaps.

Remark. The assumption that new grid lines start at the middle of a rectangle is only made for the simplicity of presentation.

We are now going to set up the finite difference scheme. For each grid point $P \in \Omega_{H}$ there are still local stepsizes $h_{P}, h_{-P}, k_{P}, k_{-P}$ corresponding to $h_{j}, h_{j-1}, k_{l}, k_{l-1}$ well-defined as the distance to the closest neighbor grid point or gridline. Hence also the weight

$$
\omega_{P}:=\frac{h_{-P}+h_{P}}{2} \frac{k_{-P}+k_{P}}{2}
$$

(see (8)) makes sense. The original scheme (4) has only to be modified in grid points, which are vertices of an exceptional triangle.

The rules for this modification are given in the following. There are four different typical orientations of exceptional triangles. We consider that one in more detail from which a new gridline in $x$-direction is starting to the right (see Fig. 2). Let $P$ be the rightmost vertex of the exceptional triangle. The two remaining vertices are $Q:=\left(x_{P}-h_{-P}, y_{P}+k_{P}\right), S:=\left(x_{P}-h_{-P}, y_{P}-k_{P}\right)$. Let $Q S$ denote the midpoint of the line segment joining $Q$ and $S$ and let $M:=\left(x_{P}-h_{-P} / 2, y_{P}\right), M Q:=\left(x_{M}, y_{M}+k_{P}\right)$, $M S:=\left(x_{M}, y_{M}-k_{P}\right)$. Modifications have only to be made in the approximation of terms containing an $x$-derivative. Firstly, consider the modified approximation in $P$. Here the simple rule is to replace after 
the formal application of (4) taken in $P$ the nonexistent grid function value $u_{H}(Q S)$ in the formula (4) by

$$
\tilde{u}_{H}(Q S):=\frac{1}{2}\left(u_{H}(Q)+u_{H}(S)\right) .
$$

The necessary modification of the scheme (4) in $Q$ and $S$ is a little bit more involved. We replace $\delta_{x} u_{H}(Q)$ in (4) by

$$
\widetilde{\delta}_{x} u_{H}(Q):=\delta_{x} u_{H}(Q)+\frac{h_{-P} k_{P}}{4 \omega_{Q}}\left(\widetilde{\delta}_{x}^{(1 / 2)} u_{H}(M)-\delta_{x}^{(1 / 2)} u_{H}(M Q)\right),
$$

where the tilde in $\widetilde{\delta}_{x}^{(1 / 2)}$ indicates that (45) has to be used where the point $Q S$ is involved. The corresponding modification in $S$ is made by taking $S$ in place of $Q$ as argument in (46). For the approximation of $\left(a u_{x}\right)_{x}$ we use

$$
\begin{aligned}
& \left.\delta_{x}^{(1 / 2)} \widehat{\left(a \delta_{x}^{1 / 2}\right.} u_{H}\right)(Q) \\
& \quad:=\delta_{x}^{(1 / 2)}\left(a \delta_{x}^{(1 / 2)} u_{H}\right)(Q)+\frac{k_{P}}{2 \omega_{Q}}\left[a(M) \widetilde{\delta}_{x}^{(1 / 2)} u_{H}(M)-a(M Q) \delta_{x}^{(1 / 2)} u_{H}(M Q)\right] .
\end{aligned}
$$

The modification in $S$ is obtained from (47) by replacing $Q$ by $S$.

It may happen that a gridpoint is the vertex of more than one exceptional triangle. Then the modification has to be repeatedly performed for each of the triangles according to the rules given above.

There may exist exceptional triangles close to the boundary $\partial \Omega$. This happens to be the case if there are points on $\partial \Omega \cap \overline{\Omega_{H}^{(r)}}$ which are not the intersection of a gridline in $x$-and in $y$-direction. In this case two of the vertices of the exceptional triangle are on $\partial \Omega$. In the remaining point a modified scheme has to be set up following the same rules as given above for the point $Q$ or $S$.

The modified operator $\widetilde{A}_{H}$ is related to a sesquilinear form $\widetilde{a}_{H}$. Let $\mathcal{T}_{H}$ be a partition satisfying condition ( $\mathrm{Rft}$ ) which is used to define the modified scheme. As in Section 4 the form $\tilde{a}_{H}$ is obtained as the sum of its values on $\Delta \in \mathcal{T}_{H}$ which we denote by $\tilde{a}_{H, \Delta}$. The present case is simpler than the one treated in Section 4 since due to the absence of the mixed derivative we need not form the mean value of two forms on different partitions as in (10). If $\Delta \in \mathcal{T}_{H}$ is a triangle in normal positions then $\tilde{a}_{H}$ is equal to $a_{H}$. So we are left with the exceptional triangles, for which we give the following definition:

$$
\begin{aligned}
& \widetilde{a}_{H, \Delta}\left(v_{H}, w_{H}\right):=a(M) \int_{\Delta}\left(P_{H} v_{H}\right)_{x}\left(P_{H} \bar{w}_{H}\right)_{x} \mathrm{~d} x \mathrm{~d} y, \\
& \widetilde{c}_{H, \Delta}\left(v_{H}, w_{H}\right):=c(Q S) \int_{\Delta}\left(P_{H} v_{I I}\right)_{y}\left(P_{H} \bar{w}_{I I}\right)_{y} \mathrm{~d} x \mathrm{~d} y, \\
& \widetilde{d}_{H, \Delta}\left(v_{H}, w_{H}\right):=P_{H}\left(d \bar{w}_{H}\right)(M) \int_{\Delta}\left(P_{H} v_{H}\right)_{x} \mathrm{~d} x \mathrm{~d} y, \\
& \tilde{e}_{H, \Delta}\left(v_{H}, w_{H}\right):=P_{H}\left(e \bar{w}_{H}\right)(Q S) \int_{\Delta}\left(P_{H} v_{H}\right)_{y} \mathrm{~d} x \mathrm{~d} y .
\end{aligned}
$$

For differently shaped exceptional $\Delta$ the definitions are similar, we do not give the details. 
The definitions of $\widetilde{A}_{H}$ and $\tilde{a}_{H}$ have been made in such a way that corresponding to Proposition 1 the relation

$$
\tilde{a}_{H}\left(v_{H}, w_{H}\right)=\left(\tilde{A}_{H} v_{H}, w_{H}\right)_{H}, \quad v_{H}, w_{H} \in \stackrel{\circ}{W}_{H},
$$

holds.

For the modified sesquilinear form $\widetilde{a}$ Theorem 2 is still valid. Hence, in the convergence proof we need only to bound the truncation error of the modified scheme. For triangles in normal position there is no difference to the expansions given in Section 5. So we consider an exceptional triangle $\Delta$. We begin with the term approximating $\left(a u_{x}\right)_{x}$. Recall the definition (45) of $\widetilde{u}(Q S)$. By Taylor expansion we derive

$$
\begin{aligned}
T_{\tilde{a}, \Delta} & :=\tilde{a}_{H, \Delta}\left(u, v_{H}\right)=a(M) \frac{u(P)-\tilde{u}(Q S)}{h_{-P}} \int_{\Delta}\left(P_{H} \bar{v}_{H}\right)_{x} \mathrm{~d} x \mathrm{~d} y \\
& =a(M) \delta_{x}^{(1 / 2)} u(M) \int_{\Delta}\left(P_{H} \bar{v}_{H}\right)_{x} \mathrm{~d} x \mathrm{~d} y+R_{\tilde{a}, 1},
\end{aligned}
$$

where

$$
\left|R_{\bar{a}, 1}\right| \leqslant \frac{k_{P}^{2}}{2 h_{-P}}\|a\|_{\infty, \Delta}\left\|u_{y y}\right\|_{\infty, \Delta} \int_{\Delta}\left|\left(P_{H} v_{H}\right)_{x}\right| \mathrm{d} x \mathrm{~d} y .
$$

The factor of $\bar{v}_{P}$ in $T_{\tilde{a}, \Delta}$, ignoring the remainder term, is given by

$$
a(M) \delta_{x}^{(1 / 2)} u(M) \frac{k_{-P}+k_{P}}{2}
$$

which gives a contribution as if $Q S$ would be a grid point and one would be dealing with a normal grid point $P$. Hence the analysis given in Section 5 applies.

The remaining part in $T_{\tilde{a}, \Delta}$ is, again ignoring the remainder term,

$$
k_{P}\left(a \delta_{x}^{(1 / 2)} u\right)(M) \frac{\bar{v}_{Q}+\bar{v}_{S}}{2}=\frac{k_{P}}{2} \bar{v}_{Q}\left(a \delta_{x}^{(1 / 2)} u\right)(M Q)+\frac{k_{P}}{2} \bar{v}_{S}\left(a \delta_{x}^{(1 / 2)} u\right)(M S)+R_{\tilde{a}, 2}+R_{\bar{a}, 3} .
$$

Here

$$
R_{\tilde{a}, 2}:=\frac{k_{P}}{2} \bar{v}_{Q}\left[\left(a \delta_{x}^{(1 / 2)} u\right)(M)-\left(a \delta_{x}^{(1 / 2)} u\right)(M Q)\right]=\bar{v}_{Q}\left[-\frac{k_{P}^{2}}{2}\left(\left(a \delta_{x}^{(1 / 2)} u\right)_{y}\right)(M)+R_{\tilde{a}, 4}\right]
$$

with $R_{\tilde{a}, 4}$ satisfying

$$
\left|R_{\tilde{a}, 4}\right| \leqslant \frac{k_{P}^{3}}{4}\left\|\left(a u_{x}\right)_{y y}\right\|_{\infty, \Delta}
$$

Similarly,

$$
R_{\tilde{a}, 3}=\bar{v}_{S}\left[\frac{k_{P}^{2}}{2}\left(\left(a \delta_{x}^{(1 / 2)} u\right)_{y}\right)(M)+R_{\tilde{a}, 5}\right],
$$

where for $R_{\tilde{a} .5}$ the same bound as for $R_{\tilde{a}, 4}$ holds. Consequently, we obtain

$$
\left|R_{\tilde{a}, 2}+R_{\tilde{a}, 3}\right| \leqslant \frac{k_{P}^{2}}{4 h_{-P}}\left[4\left\|\left(a u_{x}\right)_{y}\right\|_{\infty, \Delta} \int_{\Delta}\left|\left(P_{H} v_{H}\right)_{y}\right| \mathrm{d} x \mathrm{~d} y+\left\|\left(a u_{x}\right)_{y y}\right\|_{\infty, \Delta}|\Delta|\left(\left|v_{Q}\right|+\left|v_{S}\right|\right)\right] \text {. }
$$


The first term on the right-hand side of (50) gives the missing contribution compared to the case that $Q$ would be a normal grid point. The same observation is valid for $S$. Hence, another time the analysis in Section 5 applies.

A similar result can be proved for the approximation of $d u_{x}$. For the term in $A$ containing a $y$-derivative there is no change compared to Section 5. Hence we have the following

Proposition 4. Assume that the grids $\Omega_{H}^{(r)}$ satisfy condition $(\mathrm{Rft})$. Let $u \in C^{4}(\bar{\Omega})$. Then

$$
\tilde{a}_{H}\left(R_{H} u, v_{H}\right)=\left(R_{H}(A u), v_{H}\right)_{H}+\tilde{\tau}_{H}\left(u, v_{H}\right)
$$

with $\widetilde{\tau}_{H}\left(u, v_{H}\right)$ satisfying the estimate

$$
\begin{aligned}
\left|\widetilde{\tau}_{H}\left(u, v_{H}\right)\right| \leqslant C[ & \sum_{\Delta \in \mathcal{T}_{H}}|\Delta|(\operatorname{diam} \Delta)^{4}\left(\left\|u_{x}\right\|_{3, \infty, \Lambda}^{2}+\left\|u_{v}\right\|_{3, \infty, \Delta}^{2}\right)+\sum_{\Delta \in \mathcal{T}_{H, \mathrm{exc}}}|\Delta|(\operatorname{diam} \Delta)^{2} \\
& \left.\times\left(\sigma_{\Delta} \frac{k_{\Delta}}{h_{\Delta}}\left\|u_{x}\right\|_{2, \infty, \Delta}^{2}+\left(1-\sigma_{\Delta}\right) \frac{h_{\Delta}}{k_{\Delta}}\left\|u_{y}\right\|_{2, \infty, \Delta}^{2}\right)\right]^{1 / 2}\left\|P_{H} v_{H}\right\|_{1},
\end{aligned}
$$

where the constant $C$ is dependent of the coefficients of $A$ but not of the triangulation $\mathcal{T}_{H}$ or $u$. The quantity $\sigma_{\Delta}=1$ or 0 if a new gridline is starting from the exceptional triangle $\Delta$ in $x$-or $y$-direction, respectively.

Together with Theorem 2 this gives the proof of the following result.

Theorem 3. Assume that the conditions of Theorem 1 hold and that the grids $\Omega_{H}^{(r)}$ satisfy condition (Rft). Then the modified finite difference scheme

$$
\widetilde{A}_{H} u_{H}=g_{H} \text { in } \widetilde{\Omega}_{H}
$$

has, for $H_{\max }$ small enough, a unique solution $u_{H} \in \stackrel{\circ}{W}_{H}$ which satisfies

$$
\left\|P_{H} u_{H}-P_{H} R_{H} u\right\|_{1} \leqslant C\left|\widetilde{\tau}_{H}(u)\right|,
$$

where $\left|\widetilde{\tau}_{H}(u)\right|$ denotes the factor of $\left\|P_{H} v_{H}\right\|_{1}$ in $(51)$.

\section{Acknowledgements}

The authors gratefully acknowledge the support of this work by the Centro de Matcmática da Universidade de Coimbra-PRAXIS XXI, European project Human Capital and Mobility ERBCHRXCT:930407. The authors also thank the referee for his helpful comments and suggestions, which led to a considerable improvement in the representation of the results.

\section{References}

[1] J.A. Ferreira, Adaptive methods for parabolic problems: convergence study, Ph.D. Thesis, University of Coimbra, Portugal (1994).

[2] J.A. Ferreira, The negative norms in the supraconvergence of FDM's for two-dimensional domains, Technical Report 97-06 (1997). 
[3] P.A. Forsyth, Jr and P.H. Sammon, Quadratic convergence for cell-centered grids, Appl. Numer. Math. 4 (1988) 377-394.

[4] B. Garcia-Archila, A supraconvergent scheme for the Korteweg-de Vries equation, Numer. Math. 61 (1992) 292-310.

[5] B. Garcia-Archila and J.M. Sanz-Serna, A finite difference formula for the discretization of $\mathrm{d}^{3} / \mathrm{dx}^{3}$ on nonuniform grids, Math. Comp. 57 (1991) 239-257.

[6] R.D. Grigorieff, Some stability inequalities for compact finite difference operators, Math. Nachr. 135 (1986) 93-101.

[7] W. Hackbusch, Theory and Numerical Treatment of Elliptic Differential Equations (Springer, Berlin, 1992).

[8] F. de Hoog and D. Jackett, On the rate of convergence of finite difference schemes on nonuniform grids, J. Austrul. Math. Soc. Ser. B (1985) 247-256.

[9] H.O. Kreiss, T.A. Manteuffel, B. Swartz, B. Wendroff and A.B. White, Jr, Supraconvergent schemes on irregular grids, Math. Comp. 47 (1986) 537554.

[10] P. Lesaint and M. Zlámal, Superconvergence of the gradient of finite element solutions, RAIRO Analyse Numérique 13 (1979) 139-166.

[11] C.D. Levermore, T.A. Manteuffel and A.B. White, Jr, Numerical solutions of partial differential equations on irregular grids, in: Computational Techniques and Applications, CTAC-87, Sydney, 1987 (North-Holland, Amsterdam, 1987) 417-426.

[12] T.A. Manteuffel and A.B. White, Jr, The numerical solution of second order boundary value problems on nonuniform meshes, Math. Comp. 47 (1986) 511-535.

[13] M.A. Marletta, Supraconvergence of discretization methods on nonuniform meshes, M.Sc. Thesis, Oxford University (1988).

[14] K.W. Morton and E. Süli, Evolution-Galerkin methods and their supraconvergence, Numer. Math. 71 (1995) 331-355.

[15] E. Süli, Convergence of finite volume schemes for Poisson's equation on nonuniform meshes, SIAM J. Numer. Anal. 28 (1991) 1419-1430.

[16] A. Weiser and M.F. Wheeler, On convergence of block-centered finite differences for elliptic problems, SIAM J. Numer. Anal. 25 (1988) 351-375.

[17] B. Wendroff and A.B. White, Jr, Some supraconvergent schemes for hyperbolic equations on irregular grids, in: Nonlinear Hyperbolic Equations-Theory, Computation Methods, and Applications, Aachen, 1988, Notes on Numerical Fluid Mechanics, Vol. 24 (Vieweg, Braunschweig, 1988) 671-677.

[18] M. Zlámal, Superconvergence and reduced integration in finite element method, Math. Comp. 32 (1978) 663685. 